\title{
A Motivação de Alunos no Contexto da Progressão Continuada
}

\author{
Edna Rosa Correia Neves \\ Evely Boruchovitch \\ Universidade Estadual de Campinas
}

\begin{abstract}
RESUMO - Este trabalho teve como objetivo investigar as orientações motivacionais intrínsecas e extrínsecas de alunos no contexto da progressão continuada. A amostra foi composta de 160 estudantes de $2^{\mathrm{a}}, 4^{\mathrm{a}}, 6^{\mathrm{a}}$ e $8^{\mathrm{a}}$ séries do ensino fundamental. Os dados foram coletados mediante a apresentação de duas pranchas com histórias envolvendo a motivação intrínseca, extrínseca e a progressão continuada. Os participantes foram entrevistados individualmente. As respostas foram transcritas na íntegra e submetidas à análise de conteúdo. Os resultados indicaram que uma porcentagem expressiva de estudantes não conhecem o sistema de progressão continuada. Quanto à motivação para estudar, mesmo sabendo que não irão repetir de ano, os alunos apresentaram uma orientação motivacional predominantemente intrínseca com o avançar da idade e da escolaridade. Concluise pela importância do aprofundamento dos conhecimentos acerca do impacto da progressão continuada na motivação para aprender dos alunos.
\end{abstract}

Palavras-chave: motivação estudantil; progressão continuada; ensino fundamental.

\section{Students' Motivation in the Context of the Continuous Progression}

\begin{abstract}
This study investigated the intrinsic and extrinsic motivacional orientations of students in the context of the educational continuous progression. The sample was composed of 160 subjects of second, fourth, sixth and eighth grades of the elementary school. Data was collected by means of the presentation of histories involving the intrinsic, extrinsic motivation and the educational continuous progression system. Subjects were interviewed individually. Their answers were transcribed verbatim and submitted to content analysis. Results indicated that a expressive percentage of students did not know the educational system of continuous progression. Students revealed a predominantly intrinsic motivation orientation with advances in age and in school grade level, even though knowing that they will not repeat any school grade. This study pointed out the importance of deepening our knowledge concerning the impact of the educational continuous progression in students' motivation to learn.
\end{abstract}

Key words: student motivation; continuous progression; elementary school.

No Brasil, a preocupação com a evasão e a reprovação escolar é um problema antigo e tem merecido a atenção de educadores, psicólogos, pedagogos, psicopedagogos, sociólogos, bem como de órgãos relacionados à educação, que mediante estudos e implantação de novas propostas pedagógicas, têm procurado verificar os fatores que interferem no sucesso escolar dos alunos, visando melhorar a atual situação do ensino nacional. Nos últimos anos, medidas governamentais têm sido tomadas para reverter os problemas atribuídos ao sistema educacional, tais como: a implantação dos ciclos e a progressão continuada. O regime de Progressão Continuada, criado em 1996, é um conjunto de orientações incorporado à Lei de Diretrizes e Bases da Educação Nacional (LDB - Lei 9394/96, artigo 32, parágrafo segundo) e adotado no Ensino Fundamental pelas escolas da rede pública. Essa iniciativa governamental propõe efetuar o trabalho escolar independente das notas. Mais precisamente, isto equivale a dizer que desaparecem os tradicionais boletins e as notas bimestrais,

1 Adaptado da dissertação de mestrado da primeira autora, desenvolvida sob a orientação da segunda, e parcialmente apresentado na XXXI Reunião Anual de Psicologia da Sociedade Brasileira de Psicologia em 2001. Apoio financeiro da CAPES.

2 Endereço: Rua Paulo Vianna de Souza 270, ap. F-11, Campinas, SP, Brasil, 13060-726. E-mail: ednacneves@bol.com.br ficando apenas a avaliação formativa no interior da sala de aula com os pareceres descritivos no final de cada bimestre, sem notas ou conceitos (Fregoneze, 2000).

No estado de São Paulo, o Conselho Estadual de Educação instituiu o regime de progressão com a possibilidade do ensino fundamental ser organizado, em um ou mais ciclos. Objetiva-se com a progressão continuada um novo tratamento para o processo de avaliação na escola. Perdem sentido as expressões habituais de "aprovação" e "reprovação" e entram os conceitos de progressão, aprendizagens diferenciais e desenvolvimento global. A avaliação transforma-se então num instrumento-guia essencial para a observação da progressão do aluno (CEE 119/97). Conforme a LDB 9394/96 e o regime de progressão continuada, a avaliação do rendimento escolar do aluno deverá ser realizada periodicamente pelo professor, devendo o aluno concluir o ensino fundamental, em no máximo dez anos, podendo repetir o ano apenas no final de cada ciclo ( $4^{\mathrm{a}}$ e $8^{\mathrm{a}}$ série) e por faltas, em qualquer série.

O relatório do Conselho Estadual de Educação de São Paulo - Processo CEE No 119/97 descreve como cada escola pode se organizar e assim facilitar a implantação da progressão continuada:

O regime de progressão continuada pede avaliação continuada do processo de aprendizagem dos alunos, o qual deve ser objeto de recuperação continuada e paralela, a partir de um ciclo 
deve garantir também a progressão continuada na transição de um para outro ciclo. Enfatiza essa Deliberação, à exaustão, a necessidade de avaliações da aprendizagem, do desenvolvimento do aluno, do próprio ensino e avaliações institucionais; a necessidade das atividades de reforço e de recuperação, de meios alternativos e adaptação, reclassificação, avanço, reconhecimento, aproveitamento e aceleração de estudos, de indicadores de desempenho, controle de freqüência dos alunos e dos dispositivos regimentais adequados (CEE 119/97).

De acordo com Sousa (2001), a organização não seriada do ensino existe antes da lei 9394/96. No artigo 104 da LDB N. 4.024 de 1961 já era permitido: "Organização de cursos ou escolas experimentais com currículos, métodos e períodos escolares próprios". Na LDB N. ${ }^{\circ}$ 5.692, de 1971, artigo 14, parágrafo $4^{\circ}$, destaca-se o seguinte texto: "Verificadas as necessárias condições, os sistemas de ensino poderão admitir a adoção de critérios que permitam avanços progressivos dos alunos pela conjugação dos elementos de idade e aproveitamento". Finalmente, na LDB N 9394 de 20 de Dezembro de 1996, a ruptura da prática classificatória é recolocada de modo mais claro no artigo 23:

A educação básica poderá organizar-se em séries anuais, períodos semestrais, ciclos, alternância regular de períodos de estudos, grupos não seriados, com base na idade, na competência e em outros critérios, ou por forma diversa de organização, sempre que o interesse do processo de aprendizagem assim o recomendar (LDB No 9394: Art. 23).

A adoção desses novos critérios é uma forma de organizar os oito anos de escolaridade em períodos plurianuais, com vista a possibilitar que o aluno curse determinada série, sem interrupções. Para Borges (2001), esta nova forma de organizar o ensino deve ser dirigida pelo entendimento de que é preciso praticar a pedagogia do sucesso, ao invés da pedagogia da reprovação, percebendo no ato educativo um encontro de indivíduos e de ritmos diferentes. Por sua vez, Sousa (2001) ressalta que o sistema de avanços progressivos implica em uma adaptação dos objetivos educacionais às potencialidades de cada estudante, agrupando os alunos pela idade e avaliando o aproveitamento de cada um, em função de suas capacidades. A seguir, resultados de pesquisas relativas a progressão continuada realizadas com alunos e professores serão brevemente apresentados.

\section{Pesquisas relativas à progressão continuada}

A visão dos professores sobre o impacto das reformas no cotidiano escolar foi descrita por Fusari, Almeida, Santos, Pimenta e Manfredi (2001) tendo como referência uma pesquisa de opinião realizada pelo Sindicato de Professores do Ensino Oficial do Estado de São Paulo em 2000 com 10.027 professores. Os resultados revelaram que para a maioria dos entrevistados $(88,1 \%)$ a implantação dos ciclos e progressão continuada não ajudou a melhorar as condições de ensino e aprendizagem. Sobre as melhorias resultantes do processo de implantação das inovações, os professores apontam que a eliminação da defasagem idade/série foi o único aspecto alterado. Afirmaram, ainda, que a inexistência de reprovação entre as séries está gerando um maior desinteresse dos alunos pelos conteúdos ensinados (93,3\%). Finalmente, para 91,9\% dos professores, a promoção automática está permitindo que os alunos progridam de uma série a outra sem se apropriarem dos conteúdos fundamentais.

Uma certa resistência docente à proposta da progressão continuada e um despreparo por parte dos professores para lidar com a mesma foram encontrados nos estudos de Viégas (2002) e Carraro (2003). Os resultados da pesquisa realizada por Bertagna (2003) com 124 alunos de $1^{\mathrm{a}}$ a $4^{\mathrm{a}}$ séries e sete profissionais da área educacional parecem confirmar a presença da seletividade escolar, principalmente pela avaliação informal, sugerindo também que a implantação dos ciclos e progressão continuada não parecem ter ajudado a melhorar as condições de aprendizagem, nem ter favorecido a melhoria do rendimento escolar dos alunos.

Visando verificar o interesse, o valor dado ao estudo e os significados que a avaliação tem assumido para os estudantes de $1^{\mathrm{a}}$ a $8^{\mathrm{a}}$ séries do ensino fundamental após a implantação da progressão continuada, Arcas (2003) encontrou que os alunos expressam interesse pelo estudo independente da progressão continuada. As respostas apresentadas pelos estudantes apontam a escola como a principal forma de ascensão social e de melhoria de vida. Os dados revelaram, ainda, que a possibilidade de reprovação no interior da escola não desapareceu com a implantação da progressão continuada. Continuou existindo através do SARESP e do limite de faltas. Resultados provenientes de outras investigações com alunos são consistentes com os estudos existentes no que toca ao fato do regime de progressão continuada, possivelmente, não ter contribuído para melhorar o desempenho acadêmico dos estudantes, apontandose ainda que esta medida não parece ter sido útil para resgatar o autoconceito dos mesmos (Jacob, 2001; Jacob, Loureiro, Marturano, Linhares \& Machado, 1999).

Para Nascimento (1999), a avaliação classificatória e seletiva é um sistema que precisa e pode ser mudado, visto que, a seletividade por meio da aprovação ou da reprovação não garante a qualidade do ensino. Pode-se dizer, de acordo com Nascimento (1999), que a ruptura da seriação proposta pela progressão continuada foi um grande avanço para a educação. No entanto, pelo que apontam as pesquisas anteriormente revistas, a implantação desse regime parece não ter contribuído para melhorar o rendimento escolar dos alunos. Pode-se perceber também pela revisão de literatura realizada que os estudos sobre o sistema de progressão continuada são ainda escassos, tornando prematura qualquer conclusão definitiva acerca do real impacto desta medida para professores e alunos. Entre os estudos existentes, constata-se que a maioria diz respeito a professores (Bertagna, 2003; Carraro, 2003; Fusari \& cols., 2001; Viégas, 2002). Um menor número de investigações lidaram com as percepções dos alunos acerca do valor do estudo (Arcas, 2003) e com as auto-percepções acerca do próprio rendimento escolar (Jacob, 2001; Jacob \& cols., 1999).

\section{Teorias sócio-cognitivas da motivação para aprendizagem}

Tendo como referencial as teorias sócio-cognitivas da motivação para aprendizagem, o presente trabalho procurou 
verificar a motivação do aluno no contexto da progressão continuada. No âmbito escolar, a motivação é o fator interno que impulsiona o aluno para estudar, iniciar os trabalhos e perseverar neles até o fim. Segundo Bzuneck (2001), toda pessoa dispõe de recursos pessoais como o tempo, a energia, os talentos, os conhecimentos e as habilidades. Esses recursos poderão ser investidos em qualquer atividade escolhida pelo indivíduo, sendo mantidos, enquanto estiverem atuando os fatores motivacionais. Desta forma, a motivação pode influenciar no modo como o indivíduo utiliza suas capacidades, além de afetar sua percepção, atenção, memória, pensamento, comportamento social, emocional, aprendizagem e desempenho.

Várias pesquisas têm sido realizadas nos últimos anos, por educadores e psicólogos, sobre a motivação do aluno no contexto escolar. As cognições humanas, tais como crenças, pensamentos, expectativas e sentimentos relacionados ao processo de aprender têm sido objeto de estudo das teorias sócio-cognitivas da motivação para a aprendizagem (Dweck \& Leggett, 1988; Markus \& Nurius, 1986; Maher \& Pintrich conforme citados por Anderman \& Maher, 1994; Nicholls \& Miller, 1983,1984; Weiner, 1979). De modo geral, os resultados destas pesquisas apontam para a existência de modelos motivacionais, muitas vezes, inapropriados para o processo de aprendizagem dos indivíduos, o que incluiriam, entre outros aspectos, orientações motivacionais inadequadas (Boruchovitch, 2001).

As abordagens sócio-cognitivistas da motivação têm demonstrado a existência de duas orientações motivacionais: a intrínseca e a extrínseca. A motivação intrínseca configura-se como uma tendência natural para buscar novidades e desafios. O indivíduo realiza determinada atividade pela própria causa, por considerá-la interessante, atraente ou geradora de satisfação. É uma orientação motivacional que tem por característica a autonomia do aluno e a auto-regulação de sua aprendizagem. Já a motivação extrínseca tem sido definida como a motivação para trabalhar em resposta a algo externo à tarefa, como a obtenção de recompensas externas, materiais ou sociais, em geral, com a finalidade de atender solicitações ou pressões de outras pessoas, ou de demonstrar competências e habilidades (Amabile, Hill, Hennessey \& Tighe, 1994; Csikszentmihalyi \& Nakamura, 1989; Fortier, Vallerand \& Guay, 1995; Harackiewicks \& Elliot, 1983; Mandelink \& Harackiewicz, 1984; Sansone, 1986).

No contexto escolar, há indicadores de que a motivação intrínseca facilita a aprendizagem e o desempenho dos estudantes. $\mathrm{O}$ aluno intrinsecamente motivado envolve-se em atividades que oferecem a oportunidade para o aprimoramento de seus conhecimentos e de suas habilidades. Por sua vez, o indivíduo extrinsecamente motivado, realiza uma tarefa escolar para melhorar suas notas ou para conseguir prêmios e elogios (Guimarães, 2003). Estudos baseados na Teoria da Autodeterminação têm apresentado novas considerações sobre a motivação extrínseca, demonstrando que o comportamento extrinsecamente motivado também pode ser autodeterminado, não sendo necessariamente sempre negativo para a aprendizagem (Cokley,2000; Deci \& Ryan, 1995; Rigby, Deci, Patrick \& Ryan, 1992; Ryan \& Decy, 2000, ab; Ryan \& Stiller, 1991).

Em síntese, pode-se dizer que a motivação para a aprendi- zagem vem sendo entendida pelos teóricos contemporâneos como um constructo multidimensional caracterizado por teorias pessoais acerca da própria inteligência, atribuições de causalidade, orientações motivacionais intrínsecas e extrínsecas, metas de realização e variáveis relativas ao self como o autoconceito e a auto-eficácia do aluno. Destaca-se também, as variáveis relacionadas ao sistema educacional tais como os fatores contextuais ligados às crenças de auto-eficácia do professor, ao clima da sala de aula, à natureza da tarefa, à estrutura de sala de aula, ao caráter da avaliação, à cultura e às características da escola (Graham \& Weiner, 1996).

Tendo em vista que a motivação do aluno é um dos principais determinantes do êxito e da qualidade da aprendizagem, investigar a motivação dos alunos, principalmente, nesse momento no qual a avaliação da aprendizagem por meio de notas e repetência está sendo repensada, constituise num tema importante para a prática educacional. Em consonância, objetiva-se com o presente trabalho investigar o conhecimento e o impacto da progressão continuada nas orientações motivacionais de alunos do ensino fundamental de uma escola pública de Campinas. Pretende-se verificar também a existência de relações entre as orientações motivacionais dos participantes e variáveis como idade, gênero, série e rendimento escolar.

\section{Metodologia}

\section{Participantes}

A amostra foi composta de 160 alunos que cursavam a $2^{\mathrm{a}}$ $(n=40), 4^{\mathrm{a}}(n=40), 6^{\mathrm{a}}(n=40)$ e $8^{\mathrm{a}}(n=40)$ séries de uma escola da rede pública estadual do ensino fundamental de Campinas, provenientes de famílias de nível sócio-econômico desfavorecido. A faixa etária dos participantes variou entre sete e 16 anos, ou seja, 29\% da amostra encontrava-se entre sete e nove anos, $47 \%$ entre 10 e 13 anos e $24 \%$ entre 14 e 16 anos, sendo $50 \%$ do sexo masculino e $50 \%$ do sexo feminino. A maioria dos alunos não era repetente $(78 \%)$.

\section{Instrumentos}

Os dados foram coletados por meio de uma entrevista individual estruturada, com questões abertas, e mediante a apresentação de duas pranchas com duas histórias envolvendo a motivação intrínseca, a extrínseca e a progressão continuada. Cabe mencionar que essas duas pranchas fazem parte de um conjunto de doze pranchas desenvolvidas por Neves e Boruchovitch (Neves, 2002) para medir a motivação de alunos do ensino fundamental, tendo-se como base as principais características da motivação intrínseca e extrínseca provenientes da literatura da área (Amabile \& cols., 1994; Csikszentmihalyi \& Nakamura, 1989; Dalenberg, Bierman \& Furman, 1984; Fortier \& cols., 1995; Harackiewicks \& Elliot, 1993; Harter, 1981; Mandelink \& Harackiewickz, 1984; Sansone, 1986).

Como as histórias formuladas nas duas pranchas em questão relacionavam-se com a progressão continuada, antes da apresentação das mesmas foram realizadas duas questões para verificar o conhecimento dos alunos a respeito desse regime. As questões formuladas foram as seguintes: 1) Nessa 
escola repete-se ou não de ano? 2) Em todas as séries ou algumas séries - Quais?

Após os participantes responderem questões relativas ao conhecimento da progressão continuada, as pranchas foram apresentadas aos participantes, nas seguinte ordem: Prancha 1 - "Lino estuda muito, mesmo sabendo que se não estudar não irá repetir de ano. Você acha que ele está certo? Sim ou Não? Porque?" e Prancha 2 - "Gisele nunca gostou de estudar e está muito contente porque agora não precisa estudar e se esforçar para passar de ano. Você acha que ela está certa: Sim ou Não? Porque?".

Os dados relativos ao rendimento escolar dos alunos foram obtidos por meio da média geral das matérias de português e matemática, obtida no final do ano letivo após a coleta de dados. Para a estimativa da média geral de português e matemática, considerou-se sempre a maior média, independente da nota do aluno. Assim, se o aluno tivesse a média "A" em português e "B" em matemática, sua média geral seria " $\mathrm{A}$ ". As principais categorias das médias encontradas no estudo foram as seguintes: "A" + "A" - excelente, "A" + "B" - excelente, "B" + "B" - bom, "B" + "C" - bom, "C" + "C" - regular, "C" + "D" - regular, "D" + "D" - insatisfatório, "E" - evasão. Informações gerais relativas à história escolar dos participantes como aprovações e repetências anteriores também foram levadas em conta para uma melhor análise do desempenho escolar dos mesmos.

\section{Procedimentos}

Antes de se começar a pesquisa, propriamente dita, os instrumentos que seriam utilizados no estudo foram administrados, em 16 alunos ( $2^{\mathrm{a}}$ a $8^{\mathrm{a}}$ séries), de ambos os sexos e de idade entre sete e 16 anos. Esses alunos não fizeram parte da pesquisa, pois este procedimento funcionou como um pré-teste dos instrumentos e dos procedimentos de coleta de dados, tendo-se em vista o refinamento dos mesmos. Os resultados obtidos no estudo piloto não detectaram necessidade de revisão dos instrumentos e contribuíram para confirmar a importância de se coletar esses tipos de dados mediante entrevista individual e leitura das histórias por parte do entrevistador.

A coleta de dados ocorreu no ano de 2001 e foi feita na mesma escola onde foi realizado o estudo piloto. No início da coleta, obteve-se uma lista de todas as salas de aula de $2^{\mathrm{a}}$, $4^{\mathrm{a}}, 6^{\mathrm{a}}$ e $8^{\mathrm{a}}$ séries da escola. Os alunos foram sorteados aleatoriamente de todas as salas de aula existentes das séries em questão. Tendo-se em vista estabelecer um bom rapport com os participantes, os objetivos da pesquisa foram devidamente explicados para os alunos nas salas de aula e, individualmente, no momento da coleta de dados. Os participantes também foram assegurados de que a entrevista não valeria nota, não afetaria o desempenho acadêmico dos mesmos na escola, além de ser de caráter estritamente confidencial. Todos os participantes sorteados quiseram participar das entrevistas, sendo desnecessário o sorteio de reservas.

\section{Análise de dados}

As respostas dos alunos às pranchas foram estudadas por análise de conteúdo (Bardin, 1991; Berelson, 1952).
Foi utilizado o sistema de categorização de respostas para cada história, levando-se em conta a literatura existente na área (Weiner, 1979, 1985; Weinert \& Schneider, 1993). Foi desenvolvido um sistema de categorias que pudesse demonstrar, mais precisamente, as idéias de estudantes brasileiros de faixa etária entre sete e dezesseis anos.

Como descrito em Boruchovitch (1993) e objetivando-se maximizar a consistência da análise de dados, cada categoria foi definida operacionalmente, além de ter sido elaborado um conjunto de regras para classificação de cada resposta nas categorias existentes. Segue-se, à título de ilustração, regras de categorização e exemplos de respostas para duas categorias de justificativas apresentadas pelos participantes na prancha 1 : I) Estudo como um Valor Importante: Foram incluídas nesta categoria respostas que indicam a relevância do estudo em várias situações. Exemplos: "é importante estudar para aprender mais"; "é importante estudar para ser alguém na vida"; "tem que estudar tudo: as lições fáceis e as difíceis". II) Estudo como Desnecessário: Foram incluídas nesta categoria respostas que desvalorizam o estudo em virtude da não reprovação. Exemplos: "se a escola não reprova", "não precisa estudar"; "já que não reprova, não precisa estudar".

A consistência do processo de categorização foi avaliada mediante utilização de três juízes independentes. Em relação às duas pranchas utilizadas no presente estudo, a porcentagem de correspondência entre a pesquisadora e os juízes foi de $100 \%$. Para o tratamento dos dados, utilizou-se procedimentos de estatística descritiva e inferencial, com o Teste do Qui-Quadrado, tendo em vista a natureza categórica das variáveis investigadas.

Cabe mencionar, que foi examinada, também, a consistência interna das respostas dos participantes em relação ao instrumento completo utilizado. Procurou-se verificar se o participante que apresentava uma orientação motivacional extrínseca numa prancha relacionada à motivação extrínseca continuava com essa orientação em outras relacionadas à motivação extrínseca. O mesmo procedimento de análise foi realizado no que concerne às pranchas cujos conteúdos eram relativo à motivação intrínseca. Surgiram três categorias: (1) alta consistência, (2) moderada consistência e (3) baixa consistência. Foram classificados na primeira categoria os estudantes que foram inconsistentes entre zero e dois histórias $(87,8 \%)$, os participantes inconsistentes entre três e cinco histórias foram distribuídos na segunda categoria $(11,6 \%)$ e finalmente, na terceira categoria, os participantes inconsistentes entre seis e sete histórias $(0,6 \%)$. Pode-se concluir que a maioria dos alunos foi muito consistente em suas respostas ao longo do instrumento completo.

\section{Resultados}

\section{Conhecimento dos alunos a respeito da Progressão Continuada}

Como descrito anteriormente, antes da apresentação das pranchas foram realizadas duas questões para verificar o conhecimento dos alunos a respeito da progressão continuada. As questões formuladas foram as seguintes: 1) Nessa escola repete-se ou não de ano? 2) Em todas as séries ou algumas séries - Quais? 
Para a questão 1 - nessa escola repete-se ou não de ano? Surgiram três categorias de respostas: Não sabe $(9,4 \%)$; Sim $(88,1 \%)$ e Não (2,5\%). Para a questão 2: Em todas as séries ou algumas séries - Quais? Surgiram cinco categorias de respostas: Todas $(28,1 \%)$; Séries corretas - $4^{\mathrm{a}}$ e $8^{\mathrm{a}}$ séries $(18,8 \%)$; Confundem as séries $(21,9 \%)$; Apenas uma série correta $(10,6 \%)$ e Não sabe $(20,6 \%)$. Os resultados revelam que, provavelmente, somente $18,8 \%$ dos alunos conhecem o sistema de progressão continuada. Serão apresentadas a seguir as relações entre as questões 1 e 2 e os dados demográficos da amostra.

A Tabela 1 revela a existência de relações marginalmente significativas entre o conhecimento por parte dos alunos se na escola repete-se ou não de ano (questão 1) e a idade $\left(\chi^{2}(4)\right.$ $=11,82 ; p=0,05)$. A resposta "não sabe" foi mais frequiente entre os alunos mais novos. Já a opção "sim" tende a aumentar com o avanço da idade.

Não foram encontradas relações significativas entre a questão 1 e as variávies gênero, repetência e série escolar e entre a questão 2 e as variáveis sócio-demográficas da amostra.

De acordo com os resultados encontrados, os participantes que conhecem o sistema de progressão continuada $(18,8 \%)$ encontram-se entre aqueles que responderam "sim" $(88,1 \%)$ para a questão nessa escola repete-se ou não de ano? (questão 1). Procurou-se, então, primeiramente, separar os participantes que responderam "sim" para a questão $1 \mathrm{e}$, em seguida, relacionar os resultados apresentados por esses estudantes na questão 2, com os dados demográficos da amostra.

Conforme demonstrado na Tabela 2 , houve uma associação significativa entre a série escolar $\left(\chi^{2}(12)=75,74\right.$; $p=0,02$ ) e as respostas dos participantes à questão 2 . Ao contrário das categorias "todas" e "não sabe" as categorias "uma correta" e "séries corretas" tendem a aumentar com o avanço da escolaridade.

Tabela 1. Conhecimento dos alunos sobre repetência na escola em relação à idade

\begin{tabular}{llrrr}
\hline \multirow{2}{*}{ Variáveis } & & \multicolumn{3}{c}{ Idade (anos) } \\
\cline { 3 - 5 } & & \multicolumn{1}{c}{ 6 a 9 } & $\mathbf{1 0 ~ a ~ 1 3}$ & $\mathbf{1 4}$ a 16 \\
\hline Na escola, repete-se ou & Não Sabe & $26,9 \%$ & $5,4 \%$ & $7,3 \%$ \\
não de ano? & Sim & $69,2 \%$ & $92,5 \%$ & $90,2 \%$ \\
& Não & $3,8 \%$ & $2,2 \%$ & $2,4 \%$ \\
\hline Total & & $100 \%$ & $100 \%$ & $100 \%$ \\
\hline
\end{tabular}

Tabela 2. Porcentegem das respostas dos alunos no que concerne à questão 2 (Em todas as séries ou algumas séries - Quais?) e a série escolar.

\begin{tabular}{lrrrr}
\hline \multirow{2}{*}{ Variáveis } & \multicolumn{4}{c}{ Série } \\
\cline { 2 - 5 } & $\mathbf{2}^{\mathbf{a}}$ & $\mathbf{4}^{\mathbf{a}}$ & $\mathbf{6}^{\mathbf{a}}$ & $\mathbf{8}^{\mathbf{a}}$ \\
\hline Todas & 75 & 31 & 14 & 13 \\
Séries corretas & 0 & 5 & 29 & 47 \\
Confundem as séries & 6 & 42 & 37 & 13 \\
Uma correta & 0 & 14 & 12 & 24 \\
Não sabe & 19 & 8 & 8 & 3 \\
\hline Total & 100 & 100 & 100 & 100 \\
\hline
\end{tabular}

\section{Progressão Continuada, orientações motivacionais e dados sócio-demográficos}

No que concerne a prancha $1,93,7 \%$ dos participantes concordaram com o personagem da história. Surgiram cinco categorias de respostas: Estudo como um valor importante $(63,7 \%)$, Estudo como um meio de se evitar repetência futura (17,5\%), Estudo como uma obrigação $(9,4 \%)$, Estudo como desnecessário $(6,3 \%)$ e A escola deve reprovar o aluno $(3,1 \%)$. Pela prova do Qui-Quadrado, não foram encontradas relações significativas entre as cinco categorias encontradas na prancha 1 e as variáveis sócio-demograficas (idade, gênero, série e repetência escolar).

No entanto, analisando os dados da Tabela 3, observa-se relações significativas entre a concordância ou não dos participantes com o personagem da história, a idade $\left(\chi^{2}(2)=\right.$ $6,03 ; p=0,02)$ e a série escolar $\left(\chi^{2}(3)=6,23 ; p=0,02\right)$, na prancha 1 . A porcentagem de alunos que concordam com o garoto da história tende a aumentar com o avanço da idade e da escolaridade.

Na prancha 2, 98,1\% dos alunos discordaram do personagem da história. Foram encontradas 4 categorias de respostas: Estudo como um valor importante $(75,6 \%)$, Estudo como uma obrigação (12,5\%), Estudo como um meio de evitar repetência futura $(10,0 \%)$ e Estudo como desnecessário $(1,9 \%)$. Pela prova do Qui-Quadrado, não foram encontradas relações significativas entre as categorias encontradas na prancha 2 e as variáveis sócio-demograficas (idade, gênero, série e repetência escolar).

Os dados apresentados na Tabela 4 demonstram relações significativas encontradas entre a concordância ou não dos participantes com o personagem da história e a idade $\left(\chi^{2}(2)=15,75 ; p=0,00\right)$ na prancha 2 . Pode-se notar que apenas os alunos de sete a nove anos concordam com a garota da história.

Tabela 3. Concordância com personagem na prancha 1 em relação a idade e série

\begin{tabular}{lcrrrrrr}
\hline \multirow{2}{*}{ Variáveis } & \multicolumn{4}{c}{ Série } & \multicolumn{3}{c}{ Idade } \\
\cline { 2 - 8 } & $\mathbf{2}^{\mathbf{a}}$ & \multicolumn{1}{c}{$\mathbf{4}^{\mathbf{a}}$} & \multicolumn{1}{c}{$\mathbf{6}^{\mathbf{a}}$} & \multicolumn{1}{c}{$\mathbf{8}^{\mathbf{a}}$} & $\mathbf{6} \mathbf{a} \mathbf{1 0}$ & $\mathbf{1 0} \mathbf{1 3}$ & $\mathbf{1 4} \mathbf{a} \mathbf{1 6}$ \\
\hline Sim & $90,0 \%$ & $97,5 \%$ & $97,5 \%$ & $100 \%$ & $88,5 \%$ & $96,8 \%$ & $100 \%$ \\
Não & $10,0 \%$ & $2,5 \%$ & $2,5 \%$ & $0 \%$ & $11,5 \%$ & $3,2 \%$ & $0 \%$ \\
\hline Total & $100 \%$ & $100 \%$ & $100 \%$ & $100 \%$ & $100 \%$ & $100 \%$ & $100 \%$ \\
\hline
\end{tabular}

Tabela 4. Concordância com o personagem na prancha 2 e idade

\begin{tabular}{llccr}
\hline \multirow{2}{*}{ Variáveis } & & \multicolumn{3}{c}{ Idade Agrupada } \\
\cline { 3 - 5 } & & $\mathbf{7 ~ a ~ 9}$ & $\mathbf{1 0}$ a 13 & $\mathbf{1 4}$ a 16 \\
\hline \multirow{2}{*}{ Sim e Não } & Sim & $11,5 \%$ & $0 \%$ & $0 \%$ \\
& Não & $88,5 \%$ & $100 \%$ & $100 \%$ \\
\hline Total & & $100 \%$ & $100 \%$ & $100 \%$ \\
\hline
\end{tabular}

Não foram encontradas associações significativas entre a concordância ou não dos participantes com o personagem da história nas pranchas 1 e 2 e as variáveis gênero, repetência e rendimento escolar. 


\section{Discussão}

\section{Conhecimento dos alunos a respeito da Progressão Continuada}

O primeiro objetivo do presente estudo foi verificar o conhecimento por parte dos alunos sobre a progressão continuada. Observou-se, que os alunos mais novos conhecem pouco o sistema de progressão continuada, quando comparados com os mais velhos. Em geral, os resultados da presente investigação permitem inferir que uma porcentagem expressiva de estudantes não conhecem o sistema de progressão continuada. No entanto, numa pesquisa de opinião realizada pelo Sindicato de Professores do Ensino Oficial do Estado de São Paulo, pode-se observar professores associando a inexistência de reprovação entre as séries com o desinteresse dos alunos pelos conteúdos ensinados (Fusari \& cols., 2001). Deve-se tomar cuidado quando se relaciona problemas de desinteresse dos alunos com o sistema de progressão continuada. Segundo Arcas (2003), a implantação desse regime não parece ter gerado desinteresse dos alunos pela instituição escolar. Acresce-se que os resultados do presente estudo parecem indicar que os estudantes do ensino fundamental, podem ainda desconhecer o regime de progressão continuada.

De um modo geral, os resultados permitem inferir que o conhecimento dos alunos acerca da progressão continuada parece sofrer influências da variável série escolar, sendo o conhecimento desta medida maior entre os alunos de séries mais avançadas e mais velhos. Embora os resultados tenham revelado que a maioria dos participantes da presente pesquisa desconhece o sistema de progressão continuada, esses dados não devem ser generalizados, por se tratar de uma investigação realizada em apenas uma escola da rede pública do ensino fundamental. Ressalta-se a necessidade de que futuros estudos sejam conduzidos para um exame mais cuidadoso dos dados encontrados. Eles podem ser promissores no que diz respeito às implicações educacionais relacionadas a motivação e ao conhecimento dos alunos sobre a progressão continuada.

\section{A Progressão Continuada e as orientações motivacionais}

Foi também objetivo deste estudo verificar o impacto da progressão continuada nas orientações motivacionais dos estudantes. Como os dados revelaram que a maioria dos participantes desconhece o fato de que seria promovido de série independentemente do seu desempenho escolar, não foi possível analisar o impacto desta medida educacional na motivação dos participantes.

No entanto, as respostas relacionadas à concordância ou não dos participantes com os personagens das histórias mostraram-se interessantes do ponto de vista educacional, já que a maioria estava relacionada aos aspectos positivos e às vantagens que a aprendizagem traz ao indivíduo, sendo mais intrínsecas à medida que os alunos avançam na idade e na escolaridade. Além disso, houve uma baixa freqüência de respostas relacionadas à obrigatoriedade e à desvalorização do estudo, tais como: estudo "como meio de evitar repetência futura", "como obrigação" e "como desnecessário". As jus- tificativas apresentadas às histórias, no presente estudo, são semelhantes aos achados de Arcas (2003) confirmando que a maioria dos estudantes expressa motivação para estudar em função do papel da escola na promoção social do indivíduo. Nas duas investigações, os participantes parecem valorizar e reconhecer a importância do estudo para a vida, independente de qualquer outro fator.

Os dados obtidos na presente investigação demonstram que estudantes provenientes de uma escola pública de Campinas - SP parecem privilegiar mais a motivação intrínseca que a extrínseca. Esses resultados confirmam não só os dados encontrados por Martini (1999) e Arcas (2003) com crianças brasileiras do ensino fundamental, mas também estão na mesma direção dos achados de Zenorine (2002) com alunos universitários brasileiros. Todavia, os dados do presente estudo diferem dos resultados das pesquisas com alunos estrangeiros que apontam para uma diminuição na motivação intrínseca à medida que os indivíduos avançam na escolaridade (Harter, 1981; Graham \& Weiner, 1996; Ryan \& Deci, 2000a; Wigfield \& Guthrie, 1997). Outros estudos longitudinais que foram realizados com a intenção de investigar as orientações motivacionais dos estudantes ao longo do tempo sugerem, de modo geral, a existência de uma estabilidade na motivação intrínseca dos alunos, ao longo dos anos escolares, bem como salientam que o grau de estabilidade tende a variar em função das diferentes disciplinas (Gottfried, 1990; Gottfried, Fleming \& Gottfried, 2001). Faz-se necessário que mais estudos longitudinais sejam conduzidos em outros países, sobretudo no Brasil, para que se possa melhor compreender as características da motivação para aprender dos alunos (Boruchovitch, no prelo).

É interessante notar que as quatro investigações brasileiras citadas anteriormente, encontraram resultados semelhantes, embora tenham se utilizado de amostras e metodologias diferentes. Contudo, não se deve descartar que pode ter ocorrido, como em qualquer pesquisa que envolva seres humanos, a presença de respostas socialmente desejáveis (Blumenfeld, Pintrich \& Hamilton, 1986; Harter, 1981). No entanto, é importante ponderar que, no presente estudo, não se analisou apenas a concordância ou discordância dos participantes em relação aos personagens das histórias, o que seria mais passível de desejabilidade social, mas também foi feito um exame cuidadoso de todas as justificativas de respostas fornecidas pelos alunos. Acresce-se que foi realizada também uma análise da consistência interna das respostas dos participantes no instrumento completo. Pode-se dizer que o instrumento apresentou uma consistência interna considerável, visto que a maioria dos participantes foi muito consistente em suas respostas, ao longo do instrumento.

Um outro ponto que merece ser discutido é o fato de que os indivíduos, no presente estudo, não julgaram suas próprias atitudes. Mais especificamente, foram solicitados a concordar ou discordar de personagens de histórias hipotéticas, justificando, num segundo momento, suas respostas. Futuras pesquisas devem ser conduzidas, não só em situações reais, mas também nas que envolvam a identificação do participante com ele mesmo, ao invés de somente com personagens, como foi proposto no presente estudo.

Cabe mencionar ainda que as variáveis idade e série escolar foram as que mais se associaram significativamente às 
orientações motivacionais dos participantes. A inexistência de relações significativas entre as orientações motivacionais, o gênero, a repetência e o rendimento escolar dos participantes, encontradas na presente pesquisa, precisam ser verificadas por estudos posteriores. Pesquisas futuras devem também direcionar seus esforços para a ampliação do conhecimento acerca do impacto da progressão continuada na motivação dos alunos.

\section{Considerações Finais}

Investigações têm demonstrado que a implantação dos ciclos e da progressão continuada não parece ter sido suficiente para melhorar as condições de aprendizagem dos estudantes e, assim, resolver as verdadeiras causas da evasão e da reprovação escolar (Arcas, 2003; Bertagna, 2003; Carraro, 2003; Fusari \& cols., 2001; Jacob, 2001; Viégas, 2002). Desse modo, mudanças e inovações são esperadas para que a progressão continuada seja viável. É preciso investir em estratégias que possibilitem uma reestruturação de toda a organização escolar, tais como: tempo, espaço, avaliação, currículo, trabalho coletivo, relação professor-aluno e escolacomunidade, formação contínua dos professores e gestão escolar (Sousa, Steinvascher \& Alavarse, 2001). Embora essas mudanças pressuponham ações muito mais amplas é preciso, no entanto, criar meios para que o interesse intrínseco dos indivíduos não seja abafado gradualmente, especificamente com relação ao valor da tarefa, ao desafio, à curiosidade e à autonomia (Harter, 1981).

Acredita-se que um achado importante do presente estudo tal como das investigações de Arcas (2003) e Martini (1999) diz respeito ao fato de que os alunos se apresentaram motivados para estudar desde o início da escolaridade, reconhecendo, de modo geral, o valor da função social da escola e apresentando crenças positivas e favoráveis à aprendizagem. Assim sendo, os educadores têm como função garantir que esta motivação se mantenha, além de assegurar que os aspectos referentes à motivação intrínseca e à meta aprender sejam trabalhados em sala de aula. Há uma série de intervenções de natureza psicopedagógica que podem ser realizadas no sentido de promover a motivação para a aprendizagem e melhorar o desempenho acadêmico dos estudantes (Boruchovitch, 1994). No contexto escolar, as atividades devem ser desenvolvidas levando-se em consideração os elementos promotores da motivação intrínseca como apresentar constantemente desafios, promover curiosidade, diversificar planejamentos (Guimarães, 2001), jogos educativos e de regras, dinâmicas de grupo e outras situações motivadoras.

Os educadores devem ficar atentos ao modo como ensinam, pois não basta apenas ter conhecimento de diversas metodologias de ensino, optando por esta ou aquela. É preciso que o professor compreenda o seu próprio aluno: as características de sua personalidade, a etapa de desenvolvimento cognitivo, motor, emocional e social na qual ele se encontra (Lima, 1999). Segundo Guimarães (2001), é necessário que o professor seja sensível às necessidades internas e perspectivas pessoais do aluno e propicie, em sala de aula, um clima encorajador de iniciativa e de auto-expressão. Para que o aluno se torne um aprendiz autônomo e auto-regulado, são necessárias mudanças estruturais na escola e na sala de aula.
Em suma, os resultados assinalam à importância de se aprofundar conhecimentos acerca do impacto da progressão continuada na motivação para aprender dos estudantes (Neves \& Boruchovitch, 2001), tendo em vista o desconhecimento e a falta de informações desse novo sistema para os alunos e o desconforto e a insegurança que possivelmente tenham gerado para os professores. Faz-se necessário, portanto, examinar mais sistematicamente as variáveis que exercem impacto na motivação para aprender de alunos do ensino fundamental como as teorias pessoais acerca da própria inteligência, atribuições de causalidade, metas de realização, variáveis relativas ao self e, finalmente, as variáveis contextuais relacionadas ao sistema educacional.

\section{Referências}

Amabile, T.M., Hill, K.G., Hennessey, B.A. \& Tighe, E.M. (1994). The Work Preference Inventory Assessing Intrinsic and Extrinsic Motivation Orientation. Journal of Personality and social Psychology, 6(5), 950-967.

Anderman, E.M. \& Maher, M.L. (1994). Motivation and schooling in the middle grades. Review of Educational Research, 64(2), 287-309.

Arcas, P.H. (2003). Avaliação da aprendizagem no regime de progressão continuada: $O$ que dizem os alunos. Dissertação de Mestrado, Universidade Estadual de São Paulo, São Paulo.

Bardin, L. (1991). Análise de conteúdo. Lisboa: Edições 70, LDA.

Berelson, B. (1952). Content analysis in communications research. Glencoe: Free Press.

Bertagna, R. (2003). Progressão Continuada: Limites e possibilidades. Tese de Doutorado. Universidade Estadual de Campinas, Campinas.

Blumenfeld, P.C, Pintrinch, P.R. \& Hamilton, V.L. (1986). Children's concepts of ability, effort and conduct. American Educational Research Journal, 28(10), 95-104.

Borges, I.C.N. (2001). Avaliação na progressão continuada. Revista Psicopedagogia, 19(55), 93-97.

Boruchovitch, E. (1993). Health and illness related cognitions among brazilians students: A cross-cultural contribution. Tese de Doutorado, Universidade of Southern California, Los Angeles, USA.

Boruchovitch, E. (1994). As variáveis psicológicas e o processo de aprendizagem: Uma contribuição para a psicologia escolar. Psicologia: Teoria e Pesquisa, 10(1), 129-139.

Boruchovitch, E. (2001). Conhecendo as crenças sobre inteligência, esforço e sorte de alunos brasileiros em tarefas escolares. Revista Psicologia Reflexão e Crítica, 14(3), 461-467.

Boruchovitch, E. (no prelo). A study of causal attributions for sucess and failure in mathematics among brazilian students. Revista Interamericana de Psicologia.

Brasil, Lei 4.024, de 20 de Dezembro de 1961. Lei de Diretrizes e Bases da Educação Nacional, Brasília: 1961.

Brasil, Lei 5.692, de 11 de Agosto de 1971. Lei de Diretrizes e Bases da Educação Nacional, Brasília: 1971.

Brasil, Lei 9.394, de 20 de dezembro de 1996. Diretrizes e Bases da Educação Nacional, Brasília: 1996.

Bzuneck, J.A. (2001). A motivação do aluno: Aspectos introdutórios. Em E. Boruchovitch \& J.A. Bzuneck (Orgs.). Motivação do aluno: Contribuições da psicologia contemporânea. Petrópolis: Editora Vozes. 
Carraro, P.R. (2003). Crenças e representações dos professores sobre o construtivismo, os parâmetros curriculares nacionais $(P C N)$ e as inovações pedagógicas no contexto das diretrizes propostas para o ensino fundamental a partir da nova $L D B$. Dissertação de Mestrado, Universidade Estadual de São Paulo, São Paulo.

Cokley, K.O. (2000). Examining the validity of the academic motivation scale by comparing scale construction to selfdetermination theory. Psychological Reports, Missoula, 86(2), 560-564.

Conselho Estadual de Educação, CEE No $119 / 97$ - Avaliação $e$ Progressão Continuada - Conselheiros da Câmara de Ensino Fundamental, dezembro 1997.

Csikszentmihalyi, M. \& Nakamura, J. (1989). The dynamics of intrinsic motivation: A study of adolescents. Em C. Ames \& R. Ames (Orgs.), Research on motivation in education (pp. 249277). Goals and Cognitions, New York: Academic Press.

Dalenberg, C.J., Bierman, K.L. \& Furman, W. (1984). A reexamination of developmental changes in causal attributions, Developmental Psychology, 20(4), 575-583.

Deci, E.L. \& Ryan, R.M. (1995). Intrinsic motivation and selfdetermination in Human Behavior. New York: Plenum.

Dweck, C.S. \& Legget, E.L. (1988). A social-cognitive approach to motivation and personality. Psychological Review, 95(2), 256-270.

Fortier, M.S., Vallerand, R.J. \& Guay, F. (1995). Academic motivational and school performance: Toward a structural model. Contemporary Educational Psychology, 20(3), 257-274.

Fregoneze, G.B. (2000). Crenças de auto-eficácia de professores em situação de alterações curriculares no ensino médio, Dissertação de Mestrado, Universidade Estadual de Londrina, Londrina.

Fusari, J.C., Almeida, M.I., Santos, R., Pimenta, S.G. \& Manfredi, S.M. (2001). As reformas educacionais no Estado de São Paulo: Com a palavra os professores. Revista de Educação APEOESP, 13, 15-29.

Gottfried, A.E. (1990). Academic intrinsic motivation in young elementary school children. Journal of Educational Psychology, 82(3), 525-538.

Gottfried, A.E., Fleming, J.S. \& Gottfried, A.W. (2001). Continuity of academic intrinsic motivation from childhood through late adolescence: A longitudinal study. Journal of Educational Psychology, 93(1), 3-13.

Graham, S. \& Weiner, B. (1996). Theories and principles of motivation. Em D.C. Berliner \& R.C. Calfee (Orgs.), Handbook of Educational Psychology. New York: Simon and Schuster MacMillian.

Guimarães, S.E.R. (2001). Motivação intrínseca, extrínseca e o uso de recompensas em sala de aula. Em Boruchovitch, E. \& Bzuneck, J.A. (Orgs.), Motivação do aluno: Contribuições da psicologia contemporânea, Petrópolis: Editora Vozes.

Guimarães, S.E.R. (2003). Avaliação do estilo motivacional do professor: adaptação e validação de um instrumento. Tese de Doutorado, Universidade Estadual de Campinas, Campinas.

Harackiewicks, J.M. \& Elliot, A.J. (1993). Achievement goals and intrinsic motivation. Journal of Personality and Social Psychology, 65(5), 904-915.

Harter, S.A. (1981). New self-report scale of intrinsic versus extrinsic orientation in the classroom: Motivational and informational components. Developmental Psychology, 17(3), 300-312.
Jacob, A.V. (2001). O desempenho escolar e suas relações com autoconceito e auto-eficácia. Tese de Doutorado, Universidade de São Paulo, Ribeirão Preto.

Jacob, A.V., Loureiro, S.R., Marturano, E.M., Linhares, M.B.M. \& Machado, V.L.S. (1999). Aspectos afetivos e o desempenho acadêmico de escolares. Psicologia: Teoria e Pesquisa, 15(2), 153-162.

Lima, L.M.S. (1999). Motivação em sala de aula: A mola propulsora da aprendizagem. Em F.F. Sisto, G.C. Oliveira \& L.D.T. Fini, (Orgs.), Leituras de psicologia para formação de professores. Petrópolis: Editora Vozes.

Mandelink, G. \& Harackiewicz, J. (1984). Proximal versus distal goal setting and intrinsic motivation. Journal of Personality and Social Psychology, 47(4), 918-928.

Markus, H.M. \& Nurius, P. (1986). Possible selves. American Psychologist, 41(4), 954-969.

Martini, M.L. (1999). Atribuições de causalidade, crenças gerais e orientações motivacionais de crianças brasileiras. Dissertação de Mestrado, Universidade Estadual de Campinas, Campinas.

Nascimento, R.B. (1999). Aprovação automática: proposta pedagógica ou casuísmo político? Instrumento: Revista de Estudo e Pesquisa em Educação, 1(1), 81-85.

Neves, E.R.C. (2002). As orientações motivacionais e as crenças sobre inteligência, esforço e sorte de alunos do ensino fundamental. Dissertação de Mestrado, Universidade Estadual de Campinas, Campinas.

Neves, E.R.C. \& Boruchovitch, E. (2001). As orientações motivacionais e o conhecimento de alunos sobre a progressão continuada [Resumo]. Em Sociedade Brasileira de Psicologia (Org.), Resumos de comunicações científicas, XXXI Reunião Anual de Psicologia (p. 198). Rio de Janeiro: SBP.

Nicholls, J.G. \& Miller, A.T. (1983). The differentiation of the concepts of difficulty and ability. Child Development, 54(4), 951-959.

Nicholls, J.G. \& Miller, A.T. (1984). Reasoning about the ability of self and others: A developmental study. Child Development, 55(6), 1900-1999.

Rigby, C.S., Deci, E.L., Patrick, B.C. \& Ryan, R.M. (1992). Beyond the intrinsic-extrinsic dichotomy: Self-determination in motivation and learning. Motivation and Emotion, 16(3), 165-185.

Ryan, R.M. \& Stiller, J. (1991). The social contexts of internalization: parent and teacher influences on autonomy, motivation, and learning. Em C. Ames \& R. Ames (Orgs.), Advances in motivation and achievement. Connecticut: Jai Press Inc, 7, 115-149.

Ryan, R.M. \& Deci, E.L. (2000a). Intrinsic and extrinsic motivations: Classic definitions and new directions. Contemporary Educational Psychology, 25, 54-67.

Ryan, R.M. \& Deci, E.L. (2000b). Self-determination theory and the facilitation of intrinsic motivation, social development, and well-being. American Psychologist, 55(1), 68-78.

Sansone, C.A. (1986). Question of competence: The effects of competence and task feedback on intrinsic interest. Journal of Personality and Social Psychology, 51(5), 918-931.

Sousa, S.M.Z.L. (2001). A avaliação na organização do ensino em ciclos. Revista de Educação APEOESP, 13, 30-36.

Souza, S.M.Z.L., Steinvascher, A. \& Alavarse, O.M. (2001). Progressão continuada: Re-significando a avaliação escolar. Revista Psicopedagogia, 19, 10-14. 
Viégas, L. de (2002). Progressão continuada e suas repercussões na escola pública paulista: Concepções de educadores. Dissertação de Mestrado, Universidade Estadual de São Paulo, São Paulo.

Weiner, B.A (1979). Theory of motivation for some classroom experiences. Journal of Educational Psychology, 71(1), 3-25.

Weiner, B.A. (1985). An attributional theory of achievement motivation and emotion. Psychological Review, 92(4), 548573.

Weinert, F.E. \& Schneider, W. (1993). The munich longitudinal study on the genesis of individual competences (LOGIC). Report 9, Max Planck - Institute fur Psychologische Forschung. Munich, Germany. Manuscrito não publicado.
Wigfield, A. \& Guthrie, J.T. (1997). Relations of children's motivation for reading to the amount and breadth of their reading. Journal of Educational Psychology, 89(3), 420-432.

Zenorine, R. Da P.C. (2002). Avaliação das metas de realização e das estratégias de aprendizagem em universitários. Dissertação de Mestrado, Universidade São Francisco, Itatiba.

Recebido em 21.10.2003

Primeira decisão editorial 19.01.2004

Versão final em 29.03.2004

Aceito em 12.04.2004 